\title{
Regulation of hormone-related genes involved in adventitious root formation in sweetpotato
}

\author{
Hualin Nie $\cdot$ Sujung Kim $\cdot$ Yongjae Lee $\cdot$ Hyungjun Park $\cdot$ Jeongeun Lee $\cdot$ Jiseong Kim $\cdot$ Doyeon Kim $\cdot$ \\ Sunhyung Kim
}

Received: 7 August 2020 / Revised: 20 August 2020 / Accepted: 20 August 2020

(C) Korean Society for Plant Biotechnology

\begin{abstract}
The sweetpotatoes (Ipomoea batatas) generate adventitious roots (ARs) from cut stems that develop into storage roots and make for an important means of propagation. However, few studies have investigated the hormones involved in AR development in sweetpotato. In this study, the expression patterns of hormone-related genes involved in AR formation were identified using the transcriptome data. RNA-seq data from stems grown for 0 and 3 days after cutting were analyzed. In addition, hormone-related genes were identified among differentially expressed genes (DEGs) and filtered genes, and cluster analysis was used to characterize expression patterns by function. Most hormone-related regulated genes expressed 3 days after growing the cut stems were abscisic acid (ABA)related genes, followed by ethylene- and auxin-related genes. For ABA, the biosynthesis genes (including genes annotated to NINE-CIS-EPOXYCAROTENOID DIOXYGENASE 3 (NCED3)) and signal transduction and perception genes (including genes annotated to PROTEIN PHOSPHATASE 2Cs (PP2Cs)) tended to decrease. Expression patterns of auxin- and ethylene-related genes differed by function. These results suggest that ABA, auxin, and ethylene genes are involved in AR formation and that they may be regulated in a hormone function-dependent manner. These results contribute to the identification of hormone functions during AR formation and may contribute to understanding the mechanism of AR formation in the sweetpotato.
\end{abstract}

H. Nie $\cdot$ S. Kim $\cdot$ Y. Lee $\cdot$ H. Park $\cdot$ J. Lee $\cdot$ J. Kim $\cdot$ D. Kim S. Kim $(\bowtie)$

Department of Environmental Horticulture, University of Seoul, Seoul 02504, Republic of Korea

e-mail: pgel2006@gmail.com

Y. Lee $\cdot$ H. Park

Interdisciplinary Graduate School of Agriculture and Engineering,

University of Miyazaki, Japan
Keywords Ipomoea batatas, Sweetpotato, Adventitious root, Transcriptome, Hormone

\section{Introduction}

Adventitious roots (AR) are formed during normal development and produced in response to stresses (e.g., flooding, nutrient deprivation, wounding). In addition, AR formation from cutting stems is carried out for horticultural plant propagation (Bellini et al. 2014; Steffens and Rasmussen 2016). AR formation is triggered by external signals or intrinsic hormone, and occurs in cells neighboring vascular tissues. This development mechanism was elucidated by data accumulated from various plants such as Arabidopsis thaliana, rice, and petunia (Bellini et al. 2014; Druege et al. 2016; Liu et al. 2005; Lorbiecke and Sauter 1999). AR can be considered to progress via three phases, that is, the induction, initiation, and expression phases. Initially, plants undergo an induction phase that cell division and reprogramming are initiated by external signals (Bellini et al. 2014; Lakehal and Bellini 2019). Subsequently, ARs are formed during the root initiation phase (root meristem formation) and the expression phase (root emergence, AR primordia growth) (Dong et al. 2019; Villordon et al. 2012). Various factors including hormones, are involved in AR formation, and it has been shown the regulation of these factors depend on plant species, variety, and periods. Auxin and ethylene are known to play important roles in root formation (Lakehal and Bellini 2019), and various experiments have shown auxin is involved in cell dedifferentiation and root emergence (Liu et al. 2005; Xu et al. 2005). On the other hand, ethylene mediates the formation of aerenchyma and induces epidermal cell death to promote AR formation (Colmer et al. 2006; Steffens et al. 2006; Steffens and Sauter 2009). In addition, strigolactone, gibberellins (GAs), 
cytokinins (CKs), jasmonates (JAs), and abscisic acid (ABA) are also known to be regulated during $\mathrm{AR}$ formation (Druege et al. 2016; Lakehal and Bellini 2019; Steffens and Rasmussen 2016).

AR formation by sweetpotatoes is physiologically and economically important as it affects the development of storage roots, and thus, continues to be a topic of interest. Villordon et al. (2012) found that ethylene inhibitor treatment (1-methylcyclopropene) reduced AR count and length, and Deng et al. (2012) confirmed that AR formation was induced by $\mathrm{H}_{2} \mathrm{O}_{2}$ and inhibited by ABA. Ma et al. (2015) revealed a relationship between stem node number and $\mathrm{AR}$ formation, and presented the morphologies AR development and emergence as observed under an optical microscope. In addition, Kim et al. (2020) performed RNA-seq analysis using stems cuttings to identify the transcriptome involved in AR formation by sweetpotatoes.

The mechanism of AR formation is highly complex and involves external factors and intrinsic signals, and hormone is one of the important component of the latter. However, research on AR formation by sweetpotatoes has been limited, and thus, little is known of hormonal regulation during the process. Therefore, in this study, we investigated the regulation of hormone-related genes involved in AR formation through RNA-seq data obtained from the stem cuttings of sweetpotato (Bioproject: PRJNA610478, SRA: SRR11241425, SRR11241427) (Kim et al. 2020). The genes annotated with hormone-related genes were divided by function, and their expression patterns during AR formation were clustered. These results indicate the hormones involved in $\mathrm{AR}$ formation and their regulation at the transcriptional level.

\section{Materials and Methods}

Plant material and RNA-sequencing

Sweetpotato (cv. Annouimo) stems were cut at lengths of $\sim 30 \mathrm{~cm}$, and $\sim 12 \mathrm{~cm}$ sections were planted in pot and grown for 0 or 3 days in a growth chamber at $25 \pm 3^{\circ} \mathrm{C}$ under a $16 \mathrm{~h}$ light $/ 8 \mathrm{~h}$ dark diurnal cycle to promote $\mathrm{AR}$ formation. The samples were prepared for RNA-seq in duplicate from $\sim 12 \mathrm{~cm}$ cut stem sections planted in pot while growing for 0 or 3 days. Total RNA was extracted from cutting stems by using a modified CTAB method, and treated with DNase I (Takara Bio, Japan) to remove genomic DNA (Kim and Hamada 2005). RNA sequencing was performed as previously described Kim et al. (2020).

\section{Cluster analysis of hormone-related genes}

Differentially expressed genes (DEGs) ( $\geq 2$-fold) or filtered genes (minimum number of transcripts $\geq 100$, E-value of annotation $\leq 10-5$, identity of annotation $\geq 60$ ) were selected to investigate the regulation of hormone-related genes during AR formation. DEGs were used to determine dramatically regulated hormones during AR formation, and filtered genes were used to investigate the comprehensive regulation of genes by function among the hormones selected by DEGs regulation.

First, hormone-response genes among DEGs were identified using Gene Ontology (GO) terms such as "response to auxin", "response to brassinosteroid" in The Arabidopsis Information Resource (TAIR, http://www.arabidopsis.org/) (The Gene Ontology Consortium 2019). DEGs related to hormone functions (e.g., biosynthesis, signal transduction) were also identified. The following studies were used to select each hormone-related genes from DEGs : Auxin (Kim et al. 2018; Ljung 2013; Winkler et al. 2017), Brassinosteroids (Chung and Choe 2013; Clouse 2011), Gibberellins (Sun 2008), Cytokinins (Kieber and Schaller 2014), ABA (Fujita et al. 2009; Hauser et al. 2011; Hauser et al. 2017; Kwak et al. 2002; Nambara and Marion-poll 2005; Schweighofer et al. 2004; Yoshida et al. 2015), Ethylene (Guo and Ecker 2004; Schaller and Kieber 2002; Yoo et al. 2009), Jasmonates (Acosta and Farmer 2010; Wasternack and Hause 2013), Salicylic acid (Dempsey et al. 2011; Seyfferth and Tsuda 2014).

Second, in order to investigate the regulations of hormonerelated genes by function during $\mathrm{AR}$ formation, filtered genes were selected and were divided by function based on the studies mentioned above. The hierarchical cluster analysis of filtered genes were performed by using cluster 3.0 (http:// bonsai.hgc.jp/ mdehoon/software/cluster/software) (de Hoon et al. 2004), and heatmap was visualized by Java Treeview (http://jtreeview.sourceforge.net/) (Saldanha 2004) software.

\section{Results}

Regulations of hormone-related genes during AR formation

We performed transcriptome analysis on data obtained from cuttings stems grown for 0 and 3 days to investigate the hormones involved in AR formation. The RNA-seq showed 42,459 representative transcripts and 2,092 DEGs including genes involved in single-organism metabolic process, cell periphery, and the biosynthesis of secondary metabolites 
(Kim et al. 2020). Hormone-related genes were selected from DEGs ( $\geq 2$-fold change) and their regulations were confirmed (Fig. 1).

First, genes corresponding to each hormone response gene as indicated by the GO terms "response to auxin" and "response to BR" among DEGs were examined to identify the hormones involved in AR formation. The number of regulated hormone response genes was the highest for ABA, followed by auxin and ethylene. Genes annotated to 'response to ABA' were more likely to decrease (68 genes) than increase (33 genes). On the other hand, more genes annotated to 'response to auxin' (up: 20 genes, down: 9 genes) and 'response to ethylene' (up: 12 genes, down: 7 genes) were up-regulated. In addition, the ratios of regulated genes among genes corresponding to each GO term were calculated to investigate the regulation of these hormones during AR formation in more detail. We found $23.5 \%$ (101 genes) of 430 genes corresponding to the GO term 'response to $\mathrm{ABA}$ ' were regulated more than 2-fold.

Second, the studies on the hormone-related genes men-

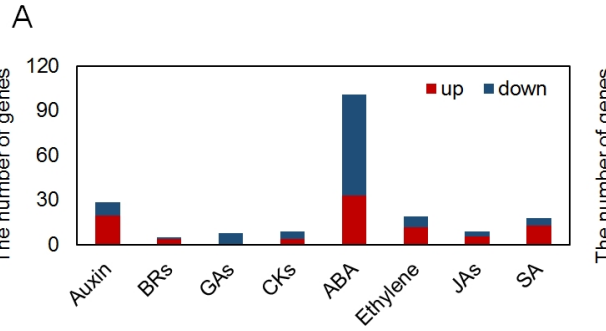

B
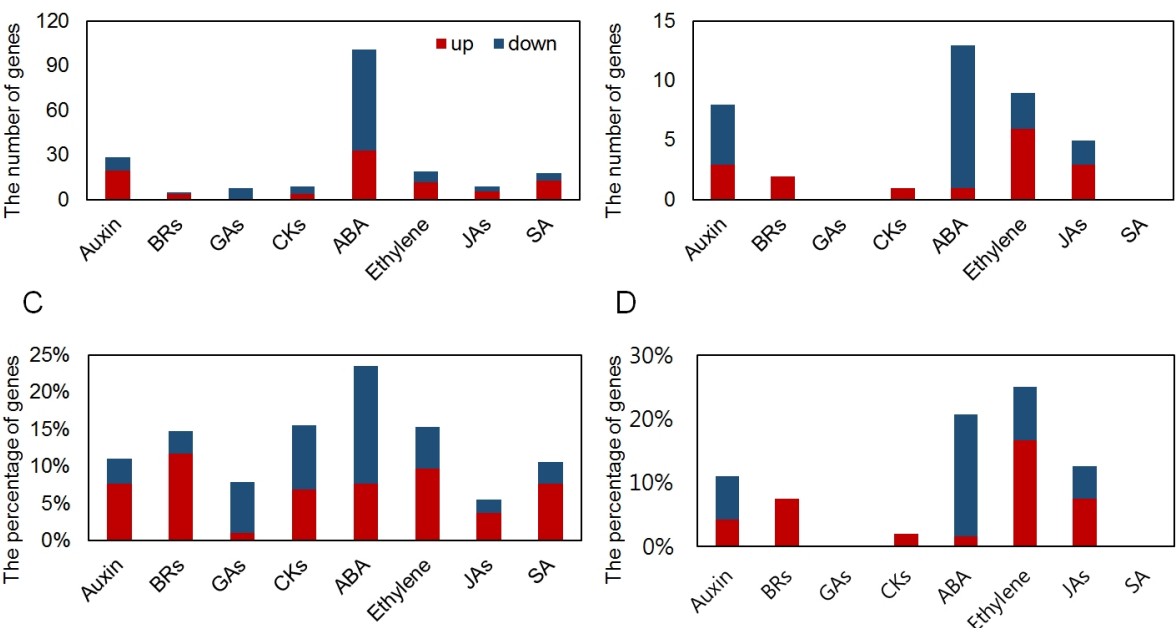

D

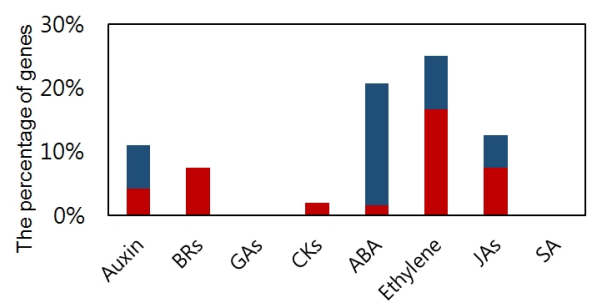

Fig. 1 Hormone regulation during adventitious root formation in sweetpotato. 'A' and 'B' show the number of differentially expressed genes (DEGs) corresponding to GO response to each hormone and the number of DEGs involved in biosynthesis and signal transduction for each hormone. ' $\mathrm{C}$ ' and ' $\mathrm{D}$ ' indicate the ratios of DEGs shown in A and B among the hormone-related genes. DEGs were filtered based on $\geq 2$-fold expressional changes. The red and blue bars indicate up- and down-regulated genes, respectively. BRs, brassinosteroids; GAs, gibberellins; CKs, cytokinins; ABA, abscisic acid; JAs, jasmonates; SA, salicylic acid

Table 1 Regulated hormone-related genes during adventitious root formation in sweetpotato. Regulated biosynthesis and signal transduction genes of each hormone were identified

\begin{tabular}{|c|c|c|c|}
\hline Hormone & Regulation & No. gene & \\
\hline \multirow[b]{2}{*}{ Auxin } & $\mathrm{Up}$ & 3 & I.bat1SL031281t004 (IAA14), I.bat1SL002990t003 (IAA3), I.bat1SL033544t001 (IAA3) \\
\hline & Down & 5 & $\begin{array}{l}\text { I.bat1SL027550t003 (IAA17), I.bat1SL000331t014 (IAA27), I.bat1SL001190t012 (IAA26), } \\
\text { I.bat1SL004871t003 (ARF4), I.bat1SL000009t013 (ARF2) }\end{array}$ \\
\hline \multirow{2}{*}{ BRs } & Up & 2 & I.bat1SL012835t007 (BAK1), I.bat1SL015442t003 (BSK5) \\
\hline & Down & 0 & \\
\hline \multirow{3}{*}{ CKs } & $\mathrm{Up}$ & 1 & I.bat1SL014914t003 (AHK1) \\
\hline & Down & 0 & \\
\hline & Up & 1 & I.bat1SL007169t001 (MPK3) \\
\hline $\mathrm{ABA}$ & Down & 12 & $\begin{array}{l}\text { I.bat1SL003385t003 (SnRK3.15), I.bat1SL030923t001 (SnRK3.16), I.bat1SL003948t006 (HAB1), } \\
\text { I.bat1SL001098t011 (HAB1), I.bat1SL001810t017(HAB3), I.bat1SL003377t002 (HAB2), } \\
\text { I.bat1SL031303t001 (SnRK3.15), I.bat1SL027489t003 (NCED3), I.bat1SL011651t022 (NCED3), } \\
\text { I.bat1SL026442t003 (SNRK2.6), I.bat1SL002661t004 (HAII), I.bat1SL002093t019 (NCED3) }\end{array}$ \\
\hline \multirow[t]{2}{*}{ Ethylene } & Up & 6 & $\begin{array}{l}\text { I.bat1SL025381t002 (ACO5), I.bat1SL044324t001 (ACO4), I.bat1SL036601t002 (ACO1), } \\
\text { I.bat1SL009004t002 (ERF1), I.bat1SL007169t001 (MPK3), I.bat1SL031061t009 (MKK9) }\end{array}$ \\
\hline & Down & 3 & I.bat1SL007764t002 (ACO4), I.bat1SL031208t002 (ACO4), I.bat1SL002212t002 (ACS6) \\
\hline \multirow{2}{*}{ JAs } & Up & 3 & I.bat1SL006234t006 (MYC2), I.bat1SL001468t011 (AOS), I.bat1SL015695t005 (AOS) \\
\hline & Down & 2 & I.bat1SL012217t002 (ACX2), I.bat1SL024318t002 (LOXO) \\
\hline
\end{tabular}

BRs, brassinosteroids; CKs, cytokinins; ABA, abscisic acid; JAs, jasmonates 
tioned in Materials and Methods were used to investigate the number of genes corresponding to biosynthesis and signal transduction pathways among DEGs. ABA biosynthesis and signal transduction genes were most regulated, and ethylene, auxin, and JA were also regulated. Ratios of regulated genes to total hormone-related genes showed trends similar to numbers of regulated genes. Among the regulated genes, ABA biosynthesis and signal transduction genes were down-regulated (Table 1). To investigate the regulations of ABA, auxin, ethylene, which involve many genes during AR formation, cluster analysis was performed by hormone function.

Transcript regulation according to the functions of 3 hormones

\section{$A B A$}

Filtered genes were represented by a heatmap to investigate the expression patterns of hormone-related genes by function. ABA-related gene expressions were generally lower in stems grown for 3 days (Fig. 2), particularly, those of ABA biosynthesis and signal transduction associated genes. For ABA synthesis-related genes, NCED3 annotated genes I.bat1SL011651t022 and I.bat1SL002093t019, which play important roles in the biosynthesis of $\mathrm{ABA}$, were downregulated. For signal transduction-related genes, down-regulations were more dramatic than up-regulations. Notably, the expressions of PP2Cs (HAB1, I.bat1SL001098t011 and I.bat1SL003948t006; HAI3, I.bat1SL001810t017) and PYRABACTIN RESISTANCE (PYR) / REGULATORY COMPONENT OF ABA RECEPTORs (RCARs) (PYL8, I.bat1SL003986t004), SNF1-RELATED PROTEIN KINASE2 (SnRK2) (SnRK2.3, I.bat1SL000264t005; SnRK2.6, I.bat1SL026442t003), and SNF1-RELATED PROTEIN KINASE3 (SnRK3) (SnRK3.16, I.bat1SL030923t001; SnRK3.15, I.bat1SL003385t003) were particularly down-regulated.
A

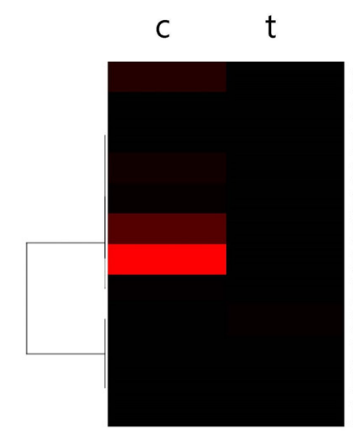

I.bat1SL027489t003; NCED3 I.bat1SL028290t005; ABA3 l.bat1SL027916t009; ABA3 l.bat1SL000337t010; ABA1 l.bat1SL009536t007; ABA1 l.bat1SL002093t019; NCED3 I.bat1SL011651t022; NCED3 l.bat1SL005489t003; ABA3 l.bat1SL018170t006; ABA2 l.bat1SL007369t005; CYP707A1 I.bat1SL041465t001; ABA2 l.bat1SL026279t001; ABA2
B

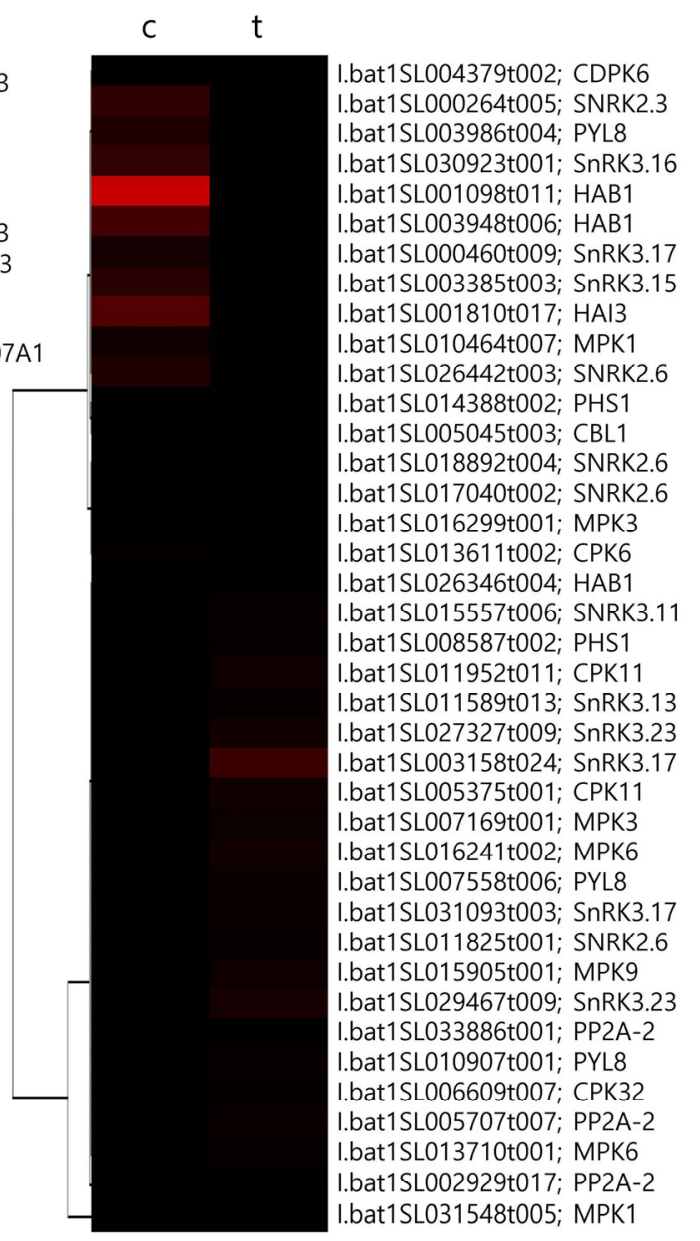

Fig. 2 Regulations of ABA-related genes on filtered genes during adventitious root formation. ' $A$ ' shows ABA biosynthesis and metabolisms genes. 'B' shows ABA signal transduction and perception genes. 'c' and ' $\mathrm{t}$ ' show stems grown for 0 and 3 days from cutting 


\section{Auxin}

The three genes involved in auxin biosynthesis were annotated, but no gene was significantly regulated during AR formation (data not shown). On the other hand, other genes (metabolism, early response, and transporter genes) were found to be regulated (Fig. 3). DIOXYGENASE FOR AUXIN OXIDATION 1 (DAO1, I.bat1SL000482t001; I.bat1SL026397t001) and IAA-LEUCINE RESISTANT 1 (ILR1, I.bat1SL011939t007), which are involved in metabolism and in the regulation of auxin activity and inactive type, were up-regulated, but GRETCHEN HAGEN3.1 (GH3.1, I.bat1SL026985t019; I.bat1 SL005912t008) and IAA-ALANINE RESISTANT3 (IAR3, I.bat1SL006780t012) were down-regulated. Regarding auxin early response genes, INDOLE-3-ACETIC ACID INDUCIBLEs (IAAs) was regulated significantly more than SMALL AUXIN UPREGULATEDs (SAURs). In particular, IAA9 (I.bat1SL 002443t012) was up-regulated and IAA27 (I.bat1SL000331t014; I.bat1SL001297t008) and IAA26 (I.bat1SL001190t012) were down-regulated. The transporter gene PIN-LIKES 5 (PILS5, I.bat1SL020724t007) was up-regulated to the greatest extent.

\section{Ethylene}

During AR formation, many ethylene-related genes were regulated, and more genes were down-regulated than upregulated (Fig. 4). Regarding ethylene biosynthesis-related genes, genes annotated to 1-AMINO-CYCLOPROPANE-1CARBOXYLIC ACID OXIDASE (ACO) were more regulated than the genes annotated to 1-AMINO-CYCLOPROPANE-1CARBOXYLATE SYNTHASE (ACS). In particular, ACO4 (I.bat1SL011932t003) and ACO1 (I.bat1SL036601t002) were up-regulated and ACO4 (I.bat1SL002350t004; I.bat1SL02 4760t004; I.bat1SL034711t003; I.bat1SL032226t001; I.bat1 SL007764t002; I.bat1SL031208t002) were down-regulated. During AR formation, regulatory mechanisms in nucleus among ethylene signal transduction genes were down-regulated, and these are the nuclear transcription activator ETHYLENEINSENSITIVE3 (EIN3; I.bat1SL002407t004, I.bat1SL012004t003, I.bat1SL000831t002) and EIN3-BINDING F BOX PROTEIN 1 (EBF1; I.bat1SL001142t002; I.bat1SL011710t001). Upregulations were slight and involved ethylene receptors (ETHYLENE RESPONSE 1 (ETR1); I.bat1SL007071t010

\section{A}

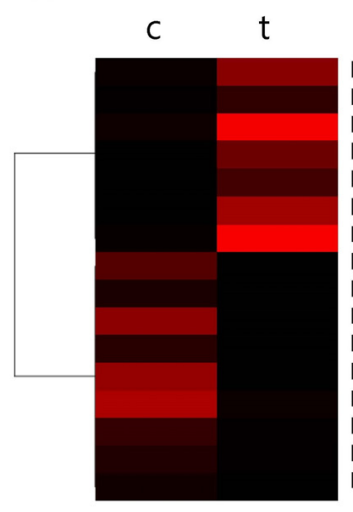

I.bat1SL026397t001; DAO1 l.bat1SL019306t002; UGT74E2 l.bat1SL000482t001; DAO1 I.bat1SL010505t007; GH3.6 l.bat1SL017535t001; IAR3 l.bat1SL009982t002; GH3.6 I.bat1SL011939t00/; ILR1 I.bat1SL006405t003; IAR3 l.bat1SL028812t001; GH3.6 I.bal1SL005912L008; GH3.1 I.bat1SL004221t006; UGT74E2 l.bat1SL006780t012; IAR3 I.bat1SL026985t019; GH3.1 l.bat1SL000210t005; IAR3 .bat1SL011986t006; IAR3 I.bat1SL029066t001; ILL3

\section{B}

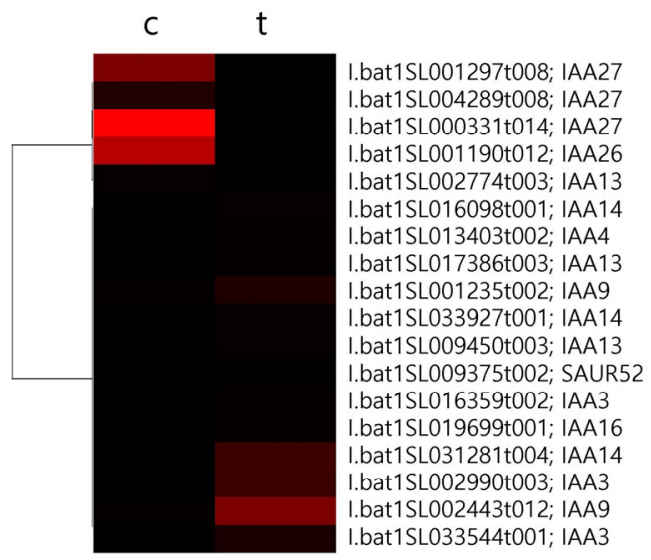

\section{C}

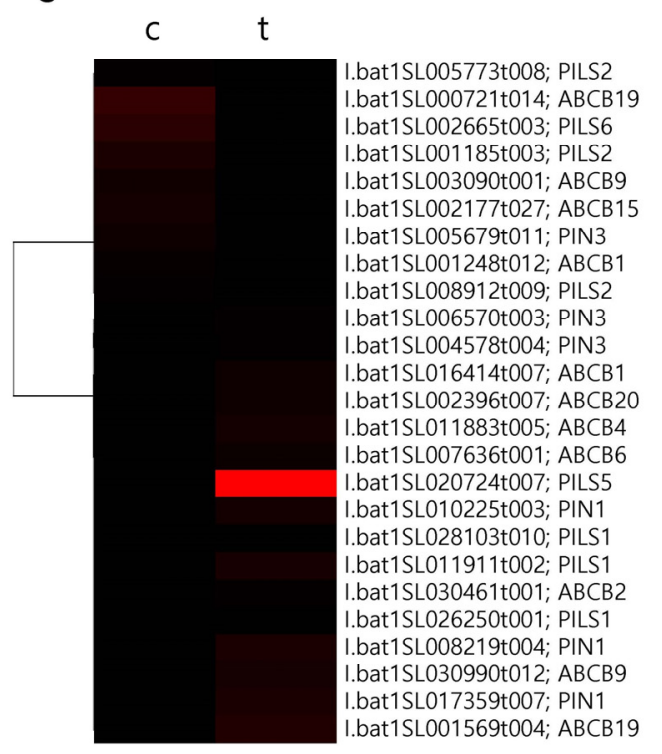

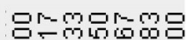

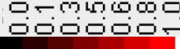

Fig. 3 Regulation of auxin-related genes on filtered genes during adventitious root formation. 'A' shows auxin metabolism-related genes. 'B' shows auxin early response genes $(I A A, S A U R)$. ' $\mathrm{C}$ ' indicates auxin transporter genes. 'c' and ' $\mathrm{t}$ ' show stems grown for 0 and 3 days from cutting 


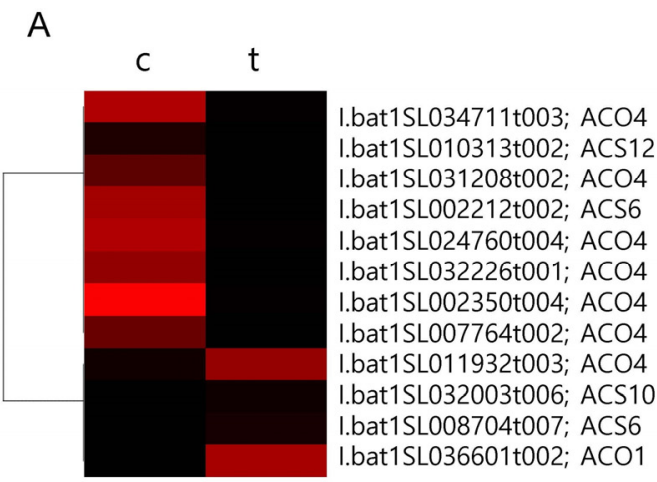

B

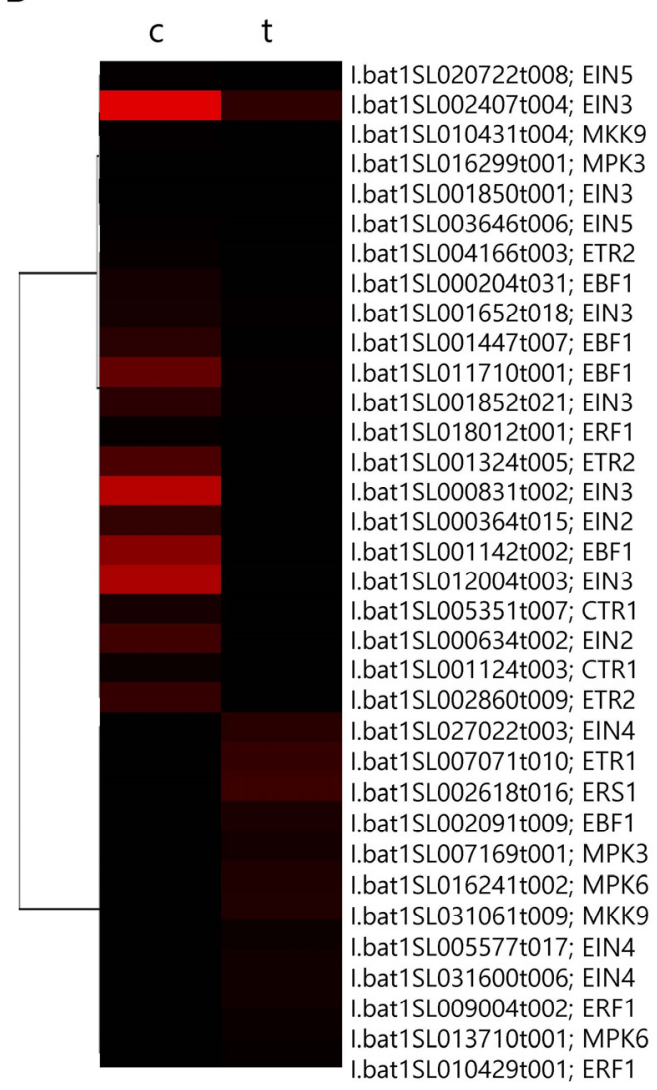

Fig. 4 Regulation of ethylene-related genes on filtered genes during adventitious root formation. 'A' shows ethylene biosynthesis and metabolisms genes. 'B' shows ethylene signal transduction genes. ' $c$ ' and ' $t$ ' show stems grown for 0 and 3 days from cutting

and ETHYLENE RESPONSE SENSOR 1 (ERS1); I.bat1 SL002618t016).

\section{Discussion}

Sweetpotatoes form ARs from stem cuttings and develop fibrous or storage roots. Since root development by sweetpotatoes directly affects productivity, several studies have addressed the subject. Recently, transcriptome analyses have been performed on sweetpotatoes at various developmental stages (Dong et al. 2019; Zhang et al. 2017). The other analysis have compared I. batatas and I. trifida to identify the genes involved in the development of tuberous roots (Ponniah et al. 2017). Research on the hormones involved in the development of sweetpotato roots have been conducted by various methods and at different development levels (McAdam et al. 2017; Nakatani and Komeichi 1991; Noh et al. 2010; Tanaka et al. 2008). On the other hand, few studies have addressed AR development from cutting stems in sweetpotato. However, recently Kim et al. (2020) conducted transcriptome analysis to investigate AR formation by sweet- potatoes and they indicated the characteristics of DEGs in AR formation of sweetpotato. In the present study, cut stems grown for 3 days were used to determine hormonerelated genes and their regulation involving in AR formation by sweetpotato. In addition, we expanded the scope of the study to hormone functions related to AR formation by using RNA-seq data and examined the regulations of hormonerelated genes during AR formation. Of these, ABA-, auxin-, and ethylene-related genes were found to be particularly regulated. We suggest that the gene expression patterns of these three hormones might provide clues regarding the types of hormones involved and their regulations during AR formation.

During AR formation, ABA-related genes were significantly down-regulated, and the expressions auxin- and ethylenerelated genes were regulated. Other hormone-related genes were also regulated, but to lesser extents. These results differ from hormone regulations observed during storage root development. For example, cytokinin expression increases during the development of tuberous roots from sweetpotato fibrous roots, and the initial expressions level of ABA- and auxinrelated genes are known to increase and then decrease 


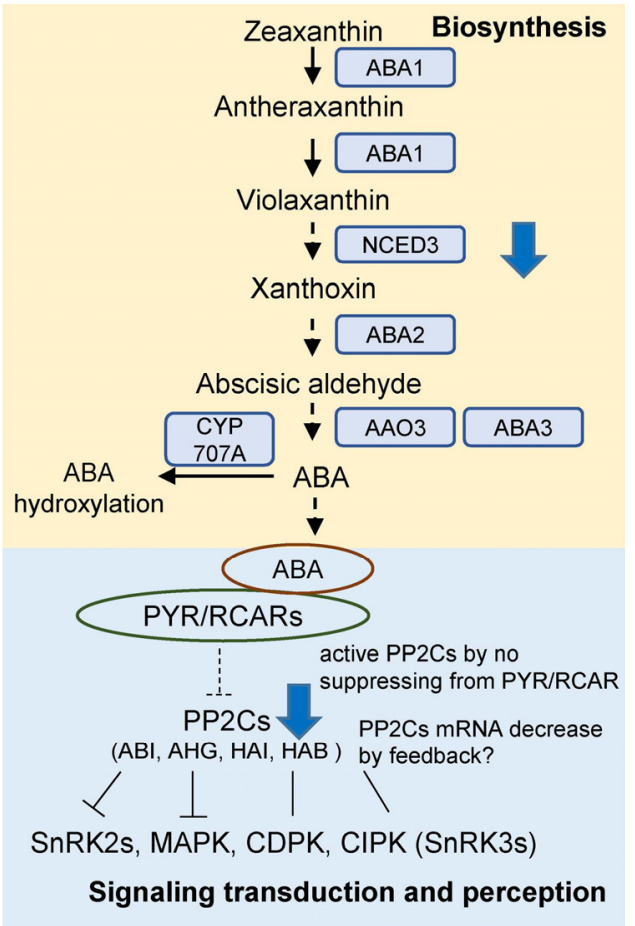

Fig. 5 Diagram of ABA-related gene regulation during adventitious root formation in sweetpotato. In the ABA biosynthesis process (yellow zone), NCED3-annotated genes expressions were particularly reduced. In signal transduction and perception (blue zone), expression of PP2Cs-annotated genes including HAB1 were reduced. Pointed arrows and flat-ended arrows represent positive interaction and negative interactions, respectively. The other lines represent currently unknown connections. Blue arrows indicate downregulated NCED3 and PP2Cs genes. The dashed lines represent the regulation expected to be reduced between NCED3 and PP2Cs genes during AR formation

again during the process (Dong et al. 2019). Other hormones have been reported to exhibit developmental level dependent expressional pattern changes (Nakatani and Komeichi 1991; Noh et al. 2010; Tanaka et al. 2008). The present study indicates that $\mathrm{ABA}$, auxin, and ethylene are probably involved in the AR formation by sweetpotatoes, but unfortunately, we did not determine details regarding their functions or crosstalk.

In order to examine the regulatory mechanisms of hormones during AR formation, hormone-related genes were classified into biosynthesis, signal transduction, metabolism, or transporter genes. The most prominent change observed in 3-day grown stems was the down-regulation of $\mathrm{ABA}$ biosynthesis genes and signal transduction genes (Figs. 2 and 5). NCED3 (I.bat1SL027489t003; I.bat1SL011651t022; I.bat1SL002093t019) is a key enzyme that catalyzes the production of xanthoxin in ABA biosynthesis (Iuchi et al. 2001), and was down-regulated during AR initiation. In addition, regarding signal transduction, the mainly regulated genes were PP2Cs (HAB1, I.bat1SL003948t006; I.bat1SL 001098t011 and HAI3, I.bat1SL001810t017) and SnRK3s (SnRK3.15, I.bat1SL003385t003; SnRK3.16, I.bat1SL030923t001). A subgroup of PP2C dephosphorylates and inactivates SnRK2 (Umezawa et al. 2009), and this activity is also regulated by $\mathrm{ABA}$ and $\mathrm{ABA}$ receptors (RCAR/PYRs). These results suggest that $A B A$ may be regulated at the transcriptional level by down-regulated NCED3, and subsequently PP2Cs, which can't be suppressed by down-regulated $\mathrm{ABA}$ and ABA-receptors (PYRs/RCARs), may be down-regulated by negative feedback during AR formation.

As described above, $\mathrm{ABA}$ was regulated at the transcription level by biosynthesis and signal transduction factors. Furthermore, these results suggest that AR formation may be induced by the down-regulation of ABA. Although Nakatani and Komeichi (1991) indicated that ABA acts on the vascular cambium of storage roots during the development of sweetpotato roots, the function of $\mathrm{ABA}$ during $\mathrm{AR}$ formation from cuttings may differ. Several studies on various plants have suggested that ABA affects AR formation. Dawood et al. (2016) showed that in Solamum dulcamara ABA inhibits $\mathrm{AR}$ formation induced by flooding, and that $\mathrm{AR}$ formation is promoted by fluridone (an inhibitor of $\mathrm{ABA}$ synthesis). In rice, $\mathrm{ABA}$ inhibits death of epidermal cells which cover root primordia during AR formation (Steffens et al. 2006; Steffens and Sauter 2009). In addition, Deng et al. (2012) showed exogenous ABA inhibits AR formation from sweetpotato cuttings via an unknown mechanism.

Auxin is known to be involved in cell dedifferentiation and $\mathrm{AR}$ emergence through a metabolism, transport, and early response gene (IAAs or SAURs) in other crops (Fig. 3) (Liu et al. 2005; Xu et al. 2005). On the other hand, in the present study, the expression of genes involved in auxin biosynthesis genes were barely regulated during AR development (data not shown), which suggests that auxin synthesized elsewhere is translocated to promote AR formation, as has been previously reported for other crops. AR formation by petunia was shown to depend on local auxin metabolism or accumulation in stems as a result of polar auxin transport (Ahkami et al. 2013). Other studies have also shown that AR formation is inhibited by auxin transport inhibitor and in OSPIN1 knockdown transgenic plants (Garrido et al. 2002; Xu et al. 2005). Therefore, the results of our study suggest AR formation by sweetpotato may be directed by auxins transported from elsewhere or converted into active forms.

Finally, it was found that ethylene also regulated the biosynthesis- and signal transduction-related genes (Fig. 4). Ethylene is known to induce epidermal cell death by $\mathrm{H}_{2} \mathrm{O}_{2}$ 
during AR formation in other plants (Steffens et al. 2006; Steffens and Sauter 2005; Steffens and Sauter 2009). Since ABA and GA have been known to be involved in this process, it would be interesting to investigate ethylene function during AR formation by sweetpotatoes and its effects on crosstalk between ABA and GA.

The AR formation of sweetpotatoes from cuttings is important, but the mechanism involved has not been elucidated. Hormone-related genes selected from RNA-seq analysis, which was performed to characterize the DEGs during AR formation by sweetpotato, were represented as heatmap by their functions. We found that ABA, auxin, and ethylene related genes were regulated during AR formation in different directions, presumably in a function dependent-manner. It would be interesting to know the functions of these hormones through further researches.

\section{Conflict of interest}

The authors have no conflict of interest to declare.

\section{Acknowledgements}

This study was funded by Project no. 117039-3 from Korea Institute of Planning and Evaluation for Technology in Food, Agriculture, Forestry and Fisheries (iPET), Ministry of Agriculture, Food and Rural Affairs

\section{References}

Ahkami AH, Melzer M, Ghaffari MR, Pollmann S, Ghorbani Javid M, Shahinnia F, Hajirezaei MR, Druege U (2013) Distribution of indole-3-acetic acid in Petunia hybrida shoot tip cuttings and relationship between auxin transport, carbohydrate metabolism and adventitious root formation. Planta 238:499-517

Acosta IF, Farmer EE (2010) Jasmonates. In The Arabidopsis Book. American Society of Plant Biologists. doi:10.1199/ tab.0129

Bellini C, Pacurar DI, Perrone I (2014) Adventitious Roots and Lateral Roots: Similarities and Differences. Annu Rev Plant Biol 65(1):639-666

Chung Y, Choe S (2013) The regulation of brassinosteroid biosynthesis in Arabidopsis. Crit Rev Plant Sci 32:396-410

Clouse SD (2011) Brassinosteroid signal transduction: From receptor kinase activation to transcriptional networks regulating plant development. Plant Cell 23:1219-1230

Colmer TD, Cox MCH, Voesenek LACJ (2006) Root aeration in rice (Oryza sativa): evaluation of oxygen, carbon dioxide, and ethylene as possible regulators of root acclimatizations. New
Phytol 170:767-777

Dawood T, Yang X, Visser EJ, Beek TA, Kensche PR, Cristescu SM, Lee S, Flokova` K, Nguyen D, Mariani C, Rieu I (2016) A Co-opted hormonal cascade activates dormant adventitious root primordia upon flooding in Solanum dulcamara. Plant Physiol 170:2351-2364

de Hoon MJ, Imoto S, Nolan J, Miyano S (2004) Open source clustering software. Bioinformatics 20:1453-1454

Dempsey DMA, Vlot AC, Wildermuth CM, Klessig FD (2011) Salicylic acid biosynthesis and metabolism. The Arabidopsis Book 9:e0156

Deng XP, Cheng YJ, Wu XB, Kwak SS, Chen W, Eneji AE (2012) Exogenous hydrogen peroxide positively influences root growth and exogenous hydrogen peroxide positively influences root growth and metabolism in leaves of sweet potato seedlings. Aust J Crop Sci 6(11):1572-1578

Dong T, Zhu M, Yu J, Han R, Tang C, Xu T, Liu J, Li Z (2019) RNA-Seq and iTRAQ reveal multiple pathways involved in storage root formation and development in sweet potato (Ipomoea batatas L.). BMC Plant Biol 19:136

Druege U, Franken P and Hajirezaei MR (2016) Plant Hormone Homeostasis, Signaling, and Function during Adventitious Root Formation in Cuttings. Front Plant Sci 7:381

Fujita Y, Nakashima K, Yoshida T, Katagiri T, Kidokoro S, Kanamori N, Umezawa T, Fujita M, Maruyama K, Ishiyama K, Kobayashi M, Nakasone S, Yamada K, Ito T, Shinozaki K, Yamaguchi-Shinozaki K (2009) Three SnRK2 protein kinases are the main positive regulators of abscisic acid signaling in response to water stress in Arabidopsis. Plant Cell Physiol 50:2123-2132

Garrido G, Ramón Guerrero J, Angel Cano E, Acosta M SánchezBravo J (2002) Origin and basipetal transport of the IAA responsible for rooting of carnation cuttings. Physiol Plant 114:303-312

Guo H, Ecker JR (2004) The ethylene signaling pathway: new insights. Curr Opin Plant Biol 7:40-49

Hauser F, Waadt R, Schroeder JI (2011) Evolution of abscisic acid synthesis and signaling mechanisms. Curr Biol 21:R346-355

Hauser F, Li Z, Waadt R, Schroeder JI (2017) SnapShot: abscisic acid signaling. Cell 171 (7):1708-1708 e1700

Iuchi S, Kobayashi M, Taji T, Naramoto M, Seki M, Kato T, Tabata S, Kakubari Y, Yamaguchi-Shinozaki K, Shinozaki K (2001) Regulation of drought tolerance by gene manipulation of 9-cis-epoxycarotenoid dioxygenase, a key enzyme in abscisic acid biosynthesis in Arabidopsis. Plant J 27:325-333

Kieber JJ, Schaller GE (2014) Cytokinins. The Arabidopsis Book 12: e0168. doi:10.1199/tab.0168

Kim SH, Hamada T (2005) Rapid and reliable method of extracting DNA and RNA from sweetpotato, Ipomoea batatas (L.). Lam. Biotechnol Lett 27:1841-1845

Kim S, Mochizuki N, Deguchi A, Nagano AJ, Suzuki T, Nagatani A (2018). Auxin contributes to the intraorgan regulation of gene expression in response to shade. Plant Physiol 177:847-862

Kim S, Nie H, Jun B, Kim J, Lee J, Kim S, Kim E, Kim S (2020) Functional genomics by integrated analysis of transcriptome 
of sweet potato (Ipomoea batatas (L.) Lam.) during root formation. Genes Genom 42:581-596

Kwak JM, Moon JH, Murata Y, Kuchitsu K, Leonhardt N, DeLong A, Schroeder JI (2002) Disruption of a guard cell-expressed protein phosphatase $2 \mathrm{~A}$ regulatory subunit, $\mathrm{RCN} 1$, confers abscisic acid insensitivity in Arabidopsis. Plant Cell 14:2849-2861

Lakehal A, Bellini C (2019) Control of adventitious root formation: insights into synergistic and antagonistic hormonal interactions. Physiol Plant 165:90-100

Liu H, Wang S, Yu X, Yu J, He X, Zhang S, Shou H Wu P (2005) ARL1, a LOB-domain protein required for adventitious root formation in rice. Plant $\mathrm{J}$ 43:47-56

Ljung K (2013) Auxin metabolism and homeostasis during plant development. Development 140:943-950

Lorbiecke R, Sauter M (1999) Adventitious Root Growth and Cell-Cycle Induction in Deepwater Rice. Plant Physiol 119(1): 21-30

Ma J, Aloni R., Villordon A, Labonte D, Kfir Y, Zemach H, Schwartz A, Althan L, Firon N (2015) Adventitious root primordia formation and development in stem nodes of 'Georgia Jet' sweetpotato, Ipomoea batatas. Am J Bot 102:1040-1049

McAdam EL, Meitzel T, Quittenden LJ, Davidson SE, Dalmais M, Bendahmane AI, Thompson R, Smith JJ, Nichols DS, Urquhart S, Gélinas-Marion A, Aubert G, Ross JJ (2017) Evidence that auxin is required for normal seed size and starch synthesis in pea. New Phytol 216:193-204

Nakatani M, Komeichi M (1991) Changes in the endogenous level of zeatin riboside abscisic acid and indole acetic acid during formation and thickening of tuberous roots in sweet potato. Jpn J Crop Sci 60:91-100

Nambara E, Marion-Poll A (2005) Abscisic acid biosynthesis and catabolism. Annu Rev Plant Biol 56:165-185

Noh SA, Lee HS, Huh EJ, Huh GH, Paek KH, Shin JS, Bae JM (2010) SRD1 is involved in the auxin-mediated initial thickening growth of storage root by enhancing proliferation of metaxylem and cambium cells in sweetpotato (Ipomoea batatas). J Exp Bot 61:1337-1349

Ponniah SK, Thimmapuram J, Bhide K, Kalavacharla V, Manoharan M (2017) Comparative analysis of the root transcriptomes of cultivated sweetpotato (Ipomoea batatas [L.] Lam) and its wild ancestor (Ipomoea trifida [Kunth] G. Don). BMC Plant Biol 17:9

Saldanha AJ (2004) Java Treeview: extensible visualization of microarray data. Bioinformatics. 20:3246-3248

Schaller GE, Kieber JJ (2002) Ethylene. In CR Somerville, EM Meyerowitz, eds, The Arabidopsis Book. American Society for Plant Biologists. pp 1-2

Schweighofer A, Hirt H, Meskiene I (2004) Plant PP2C phosphatases: emerging functions in stress signaling. Trends Plant Sci 9:236-243

Seyfferth C, Tsuda K (2014) Salicylic acid signal transduction: the initiation of biosynthesis, perception and transcriptional reprogramming. Front Plant Sci 5:697
Steffens B, Sauter M (2005) Epidermal cell death in rice (Oryza sativa L.) is regulated by ethylene, gibberellin and abscisic acid. Plant Physiol 139:713-721

Steffens B, Sauter M (2009) Epidermal cell death in rice is confined to cells with a distinct molecular identity and is mediated by ethylene and $\mathrm{H} 2 \mathrm{O} 2$ through an autoamplified signal pathway. Plant Cell 21:184-196

Steffens B, Rasmussen A (2016) The physiology of adventitious roots. Plant Physiol 170:603-617

Steffens B, Wang J, Sauter M (2006) Interactions between ethylene, gibberellin and abscisic acid regulate emergence and growth rate of adventitious roots in deepwater rice. Planta 223:604-612

Sun TP (2008) Gibberellin metabolism, perception and signaling pathways in Arabidopsis. In The Arabidopsis Book. American Society of Plant Biologists. doi:10.1199/tab.0103

Tanaka M, Kato N, Nakayama H, Nakatani M, Takahata Y (2008) Expression of class 1 Knottedl-like homeobox genes in the storage roots of sweetpotato (Ipomoea batatas). J Plant Physiol 165:1726-1735

The Gene Ontology Consortium (2019) The Gene Ontology Resource: 20 years and still GOing strong. Nucleic Acids Res 47:D330-D338

Umezawa T, Sugiyama N, Mizoguchi M, Hayashi S, Myouga F, Yamaguchi-Shinozaki K, Ishihama Y, Hirayama T, Shinozaki K (2009) Type 2C protein phosphatases directly regulate abscisic acid-activated protein kinases in Arabidopsis. Proc Natl Acad Sci USA 106(41):17588-17593

Villordon A, Clark C, LaBonte D, Firon N (2012) 1-Methylcyclopropene has a variable effect on adventitious root emergence from cuttings of two sweetpotato cultivars. Hortscience 47(12):1764-1767

Wasternack C, Hause B (2013) Jasmonates: biosynthesis, perception, signal transduction and action in plant stress response, growth and development. An update to the 2007 review in Annals of Botany. Ann Bot (Lond) 111:1021-1058

Winkler M, Niemeyer M, Hellmuth A, Janitza P, Christ G, Samodelov SL, Wilde V, Majovsky P, Trujillo M, Zurbriggen MD, Hoehenwarter W, Quint M, Calderon Villalobos LIA (2017) Variation in auxin sensing guides AUX/IAA transcriptional repressor ubiquitylation and destruction. Nat Commun 8:15706

Xu M, Zhu L, Shou H, Wu P (2005) A PIN1 family gene, OsPIN1, involved in auxin-dependent adventitious root emergence and tillering in rice. Plant Cell Physiol 46:1674-1681

Yoo SD, Cho Y, Sheen J (2009) Emerging connections in the ethylene signaling network. Trends Plant Sci 14:270-279

Yoshida T, Mogami J, Yamaguchi-Shinozaki K (2015) Omics approaches toward defining the comprehensive abscisic acid signaling network in plants. Plant Cell Physiol 56:1043-1052

Zhang K, Wu Z, Tang D, Luo K, Lu H, Liu Y, Dong J, Wang X, Lv C, Wang J (2017) Comparative transcriptome analysis reveals critical function of sucrose metabolism related-enzymes in starch accumulation in the storage root of sweet potato. Front Plant Sci 8:914 